\title{
The Non-Relativistic Coulomb Problem on a Cone
}

\author{
Gary W. Gibbons, Fernando Ruiz Ruiz ${ }^{\star}$ and Tanmay Vachaspati
}

Department of Applied Mathematics and Theoretical Physics, University of Cambridge, Silver Street, Cambridge CB3 9EW, UK

\begin{abstract}
We study the non-relativistic Coulomb problem on a cone. The non-trivial topology of the cone breaks the symmetry associated with the conservation of the Lagrange-Laplace-Runge-Lenz vector. Classically this translates into a precession of the orbits, and quantum-mechanically into a splitting of the energy levels. For the scattering problem we find that classical multi-scattering is possible and that it gives rise to a wake structure; we also evaluate the full quantum wave function and from it recover the classical results.
\end{abstract}

\section{Introduction}

There has been considerable interest recently in the problem of scattering, both classical and quantum, by a cone [1-3], that is the behaviour of geodesics or solutions of the Klein-Gordon or Schrödinger equation in the background metric

$$
d s^{2}=-d t^{2}+d z^{2}+d r^{2}+r^{2} d \phi^{2},
$$

where

$$
-\pi \alpha \leqq \phi \leqq \pi \alpha \equiv \pi-\frac{\delta}{2}
$$

and $\delta$ is the deficit angle of the cone. The purpose of the present paper is to extend this work to the case when a Coulomb potential of the form

$$
V=-\frac{K}{r}
$$

is present. Such a term may arise in a number of contexts including:

1. The induced electrostatic repulsion experienced by a particle with electric charge

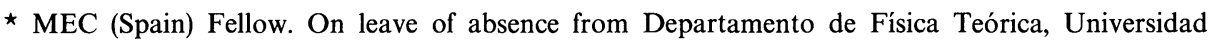
Complutense, Madrid
} 
$e$ brought up to a cosmic string [4], the constant $K$ in (1.2) being given, for small deficit angles, by

$$
K \simeq-\frac{\pi}{32} \delta e^{2}
$$

This effect may be important when considering the Callan-Rubakov effect for cosmic strings [5].

2. The induced gravitational attraction experienced by a particle of mass $m$ when brought up to a cosmic string [6], the constant $K$ in (1.2) being given, for small deficit angles, by

$$
K \simeq \frac{\pi}{32} G m \delta
$$

This effect is specially interesting because the metric outside a cosmic string is locally flat and the usual Newtonian potential $U=\frac{1}{2} \ln \left(-g_{00}\right)$ is constant. Nevertheless there is an induced attraction which, since $\delta=8 \pi G T, T$ being the energy per unit length of the string, is second order in Newton's constant $G$.

3. The slow scattering of two parallel Nielsen-Olesen vortices in the critical case when the Higgs and vector masses are equal has been recently shown to be describable as a geodesic motion on a curved "moduli space" which has the geometry (for large separations) of a flat cone with deficit angle $\delta=\pi[7,8]$. If the masses are not equal there will be forces between the vortices. The nature of these forces is not well understood but one might anticipate Coulomb-like terms.

4. More speculatively, recent work on quantum gravity in $2+1$ dimensions has involved quantum scattering on cones $[1,9]$. In this connection the induced second order attraction described may be of interest.

One final motivation for studying the problem is that we have an exactly solvable problem which allows a detailed investigation of the interaction between the so called "topological" effects due to the conical boundary condition with the long range scattering effects due to the Coulomb term. For example, it is well known that the conventional Coulomb problem exhibits "hidden" or "dynamical" symmetries which lead to degeneracies in the spectrum. We are able to study in detail how these degeneracies are split by the topological effects.

In what follows we shall mainly be concerned with the classical or quantum non-relativistic motion. For ultrastatic metrics like (1.1) (i.e. with $g_{00}=$ constant, $\left.g_{0 i}=0\right)$ and no potential term the relativistic motion does not differ significantly from the non-relativistic motion. Now, if potential terms are present this is no longer true. For example, even without the cone, special relativistic effects cause a precession of the perihelion for Keplerian ellipses. However, in the applications we have in mind it is unlikely that the simple Coulomb-like term (1.2) would remain valid if the motion were significantly relativistic. For this reason we will merely mention "en passant" the effects of special relativity. We hope in a future paper to consider the effects of spin.

The plan of the paper is as follows. In Sect. 2 we shall consider the bound 
states, for which the constant in (1.2) is positive. Classically-projected on the $(r, \phi)$ plane-we obtain precessing ellipses with one focus the vertex of the cone. The conical geometry implies that if $\delta \neq 0$ the classical Lagrange-Laplace-RungeLenz vector ceases to be well defined. Quantum-mechanically we obtain, by separation of variables in cylindrical coordinates, the spectrum of bound states and show how the conical geometry breaks the dynamical $S O(3)$ symmetry down to $O(2)$. In Sect. 3 we consider the choice of an appropriate incoming state for the scattering in the absence of a Coulomb term and comment on its related wake structure. Section 4 contains our treatment of the Coulomb scattering, both classical and quantum. As is well known, the long range nature of the Coulomb potential leads to a divergence in the differential cross-section in the forward direction. We find that in the conical case the divergence persists but is shifted by the deficit angle. Finally, Sect. 5 contains our conclusions.

\section{Bound States}

2.1 Classical Bounded Motion. We shall be concerned with the motion of a particle of mass $\mu$ in the potential (1.2). The most direct way to obtain the classical orbits is probably using the Hamilton-Jacobi method. We have

$$
\mu \frac{d x^{\alpha}}{d \lambda}+A^{\alpha}=g^{\alpha \beta} \frac{\partial S}{\partial x^{\beta}}
$$

where $A^{\alpha}=-\delta_{0}^{\alpha} K / r$ and

$$
g^{\alpha \beta} \frac{\partial S}{\partial x^{\alpha}} \frac{\partial S}{\partial x^{\beta}}=-\mu^{2} .
$$

Making the Ansatz (ignoring the $z$-motion)

$$
S=p^{0} t+j \phi+W(r)
$$

we find that

$$
\mu^{2}\left(\frac{d r}{d \lambda}\right)^{2}+j^{2} \mu^{2} \frac{1}{r^{2}}=\left(p^{0}+\frac{K}{r}\right)^{2}-\mu^{2}
$$

and

$$
r^{2} \frac{d \phi}{d \lambda}=j
$$

Defining $u=1 / r$ allows us to deduce that

$$
\left(\frac{d u}{d \phi}\right)^{2}+u^{2}=\frac{1}{j^{2} \mu^{2}}\left[\left(p^{0}\right)^{2}-\mu^{2}+2 K p^{0} u+K^{2} u^{2}\right] .
$$

The solutions of (2.1) are

$$
u=\frac{K p^{0}}{j^{2}-K^{2}}+\sqrt{\frac{\left(p^{0}\right)^{2}-\mu^{2}}{j^{2}}+\frac{K^{2}\left(p^{0}\right)^{2}}{j^{2}\left(j^{2}-K^{2}\right)}} \cos \left[\sqrt{1-\frac{K^{2}}{j^{2}}}\left(\phi-\phi_{0}\right)\right]
$$


If $K>0$, i.e. the force is attractive, and $\left(p^{0}\right)^{2}<\mu^{2}$ then $u$ will be bounded below by a positive number and hence $r$ will be bounded above. These are the classical bounded motions. They are periodic in $\phi$ with period

$$
\frac{2 \pi}{\sqrt{1-\frac{K^{2}}{j^{2}}}}
$$

Since $\phi$ is periodic with period $2 \pi \alpha$ the orbits are not in general closed, rather they are rosette shaped - the periapsis advances by an amount

$$
\Delta \phi=\delta+2 \pi\left(\frac{1}{\sqrt{1-\frac{K^{2}}{j^{2}}}}-1\right)
$$

per revolution.

In the non-relativistic limit we set $p^{0}=\mu+E$ and expand in inverse powers of the velocity of light (which has been set to unity above). The orbit is now given by

$$
u=\frac{K \mu}{j^{2}}+\sqrt{\frac{2 \mu E}{j^{2}}+\frac{K^{2} \mu^{2}}{j^{2}}} \cos \left(\phi-\phi_{0}\right)
$$

and the advance of the periapsis now becoming simply given by $\Delta \phi=\delta$, as one might have supposed without calculations.

If the motion is relativistic we also have bounded motions with $K^{2}>j^{2}$ which start from and finish at the vertex. We shall not discuss them further here.

It is well known that the existence of closed orbits in the non-relativistic flat space Kepler problem is associated with the existence of an extra conserved quantity, the Lagrange-Laplace-Runge-Lenz vector, discovered originally by Lagrange [10]. It is thus of some interest to enquire about the fate of this vector in the case that $\delta \neq 0$ and the orbits do not close. In the flat coordinates

$$
\vec{x}=(r \cos \phi, r \sin \phi)
$$

the equation of motion is

$$
\mu \ddot{\vec{x}}=-\frac{K}{x^{3}} \vec{x} .
$$

The conserved angular momentum vector points along the $z$-axis and has the value

$$
L_{z}=\mu \vec{x} \times \dot{\vec{x}} .
$$

The Lagrange-Laplace-Runge-Lenz vector $\vec{M}$ is given by

$$
\vec{M}=\dot{\vec{x}} \times \vec{L}-K \frac{\vec{x}}{|\vec{x}|},
$$

and hence lies in the $\vec{x}$ plane. Now if we pass to the cone, Eqs. (2.4), (2.5), (2.6), remain true locally and hence formally $\vec{M}$ is still conserved. However, unlike $L_{z}$ which is a scalar and thus well defined on the cone, the components of $\vec{M}$ are no 
longer globally well-defined functions on the cone (they are periodic and nonconstant with period $2 \pi$ in $\phi$ rather than $2 \pi \alpha$ ). Thus their formal conservation no longer guarantees the closure of the orbit.

Similar remarks apply to Hamilton's equivalent but less well known formulation of the extra symmetry in terms of the "hodograph." The hodograph of a motion $\vec{x}=\vec{x}(t)$ in $\mathbb{R}^{2}$ is the associated curve in velocity space $\vec{v}=\vec{v}(t)$. For Kepler motion Hamilton [11] observed that the hodograph is, in general, a circle with its centre displaced from the origin. The reader may easily establish this property using $\vec{M}$. However, the very definition of the hodograph depends on being able to parallel transport the tangent vector to the orbit to some fixed point in $\mathbb{R}^{2}$. For flat Euclidean space this parallel transport is unambiguous. On the cone, however, the holonomy is non-trivial: it depends on how one encircles the vertex. The inability to define a global Lagrange-Laplace-Runge-Lenz vector is analogous to the inability to define total momentum or total supercharge in an asymptotically conical background $[12,13]$.

2.2 Quantum-Mechanical Bound States. From a quantum-mechanical point of view one is interested in finding the bound states of the system, which amounts to solving the eigenvalue equation $H \psi=E \psi$, or equivalently

$$
\left[\frac{1}{r} \frac{\partial}{\partial r}\left(r \frac{\partial}{\partial r}\right)+\frac{1}{r^{2}} \frac{\partial^{2}}{\partial \phi^{2}}+\frac{2 \mu K}{r}-k^{2}\right] \psi_{\alpha}(r, \phi)=0,
$$

where we have introduced the notation $k^{2} \equiv-2 \mu E$. The boundary conditions on the wave-function $\psi$ are, on the one hand, the standard requirements of finiteness at the origin and convergence to zero at infinity, and on the other, that $\psi$ has to be periodic in $\phi$ with period $2 \pi \alpha$,

$$
\psi_{\alpha}(\phi)=\psi_{\alpha}(\phi+2 \pi \alpha)
$$

To solve Eq. (2.7) we make the Ansatz,

$$
\psi_{\alpha}(r, \phi)=\frac{1}{\sqrt{2 \pi \alpha}} e^{i m \phi / \alpha} R(r), \quad m=0, \pm 1, \pm 2, \ldots,
$$

which already takes care of condition (2.8). Substituting Eq. (2.9) into (2.7) and solving the resulting differential equation for $R(r)$ we obtain that the only solution which satisfies the required boundary conditions at the origin and infinity is

$$
R(r)=\frac{1}{\sqrt{r}} W_{Q,|m| / \alpha}(2 k r)
$$

where $W_{\lambda, \beta}(z)$ is Whittaker's function, and $Q \equiv \mu K / k$ and $|m| / \alpha$ are related by

$$
\frac{|m|}{\alpha}=Q-l-\frac{1}{2}, \quad l=0,1,2, \ldots .
$$

From this last equation it follows that the energies of the bound states are 


$$
E_{l m}(\alpha)=-\frac{\mu K^{2}}{2\left(l+\frac{|m|}{\alpha}+\frac{1}{2}\right)^{2}}, \quad m=0, \pm 1, \pm 2, \ldots, \quad l=0,1,2, \ldots
$$

When condition (2.10) holds $W_{Q,|m| / \alpha}(2 \mathrm{kr})$ can be written in terms of the Laguerre functions $L_{l}^{2|m| / \alpha}(2 k r)$ :

$$
R_{l m}(r)=\left[\frac{2 k^{3}}{\mu K} \frac{l !}{\Gamma\left(2 \frac{|m|}{\alpha}+l+1\right)}\right]^{1 / 2}(-1)^{l}(2 k r)^{|m| / \alpha} e^{-k r} L_{l}^{2|m| / \alpha}(2 k r),
$$

where we have introduced a normalization constant so that

$$
\int_{0}^{2 \pi \alpha} d \phi \int_{0}^{\infty} r d r \psi_{\alpha, l^{\prime} m^{\prime}}(r, \phi) \psi_{\alpha, l m}(r, \phi)=\delta_{l l^{\prime}} \delta_{m m^{\prime}}
$$

In order to have a well-defined Schrödinger eigenvalue problem the hamiltonian has to be self-adjoint. This amounts to specifying its domain, that is, the space of functions on which it acts as a self-adjoint operator. In general, this domain will not be unique. For example, in the case of the cone without potential there is a whole one-parameter family of them [14], corresponding to different behaviours of the wave function at the origin. In our case, the conditions of boundedness at the origin and vanishing at infinity ensures that the hamiltonian is self-adjoint on the function space spanned by $\psi_{\alpha, l m}(r, \phi)$. This follows from the fact that condition (2.10) implies that

$$
\int_{0}^{\infty} r d r R_{l m}(r)\left[\frac{1}{r} \frac{d}{d r} r \frac{d}{d r} R_{l^{\prime} m}(r)\right]=\int_{0}^{\infty} r d r R_{l^{\prime} m}(r)\left[\frac{1}{r} \frac{d}{d r} r \frac{d}{d r} R_{l m}(r)\right] .
$$

If the deficit angle is zero we have $\alpha=1$, which corresponds to the plane; then the energy levels only depend on the quantum number $n=l+|m|$ and have degeneracy $2 n+1$. This has to do with the fact that, apart from $H$, there are three conserved quantities, namely the angular momentum and the two components of the Lagrange-Laplace-Runge-Lenz vector. The Poisson algebra for bound states is $S O(3)$. The bound states are degenerate and carry the spin $n$ representation of $S O(3)$. However, when $\alpha \neq 1$, this symmetry breaks down and we are left with a residual $O(2)$ symmetry since now the only conserved quantity is the angular momentum, whose quantum number is $m$. The splitting of the levels for small $\delta$ can be found by expanding (2.11) in powers of $\delta$,

$$
\begin{gathered}
E_{n, m}=-\frac{\mu K^{2}}{2\left(n+\frac{1}{2}\right)^{2}}\left[1-\frac{|m|}{n+\frac{1}{2}} \frac{\delta}{\pi}+O\left(\delta^{2}\right)\right], \\
n=0,1,2, \ldots, \quad m=-n,-n+1, \ldots, n .
\end{gathered}
$$

Before closing this section let us make one more comment. Speaking of bound states only makes sense for attractive potentials $(K>0)$. This is precisely the case of the gravitationally induced potential by cosmic strings mentioned in the introduction, for which $K$ is quadratic in Newton's constant $G$. This amounts to saying that we are dealing with quantum gravitational effects quadratic in $G$. 


\section{Choice of Incoming State for Scattering}

We now turn to the problem of the scattering on a cone with and without a Coulomb potential. It will be convenient to discuss the problem in the "wedge" representation of the cone as described by Eq. (1.1).

To study the classical scattering without a Coulomb potential, consider a particle approaching the cone from the $\phi=0$ direction. Hence, in the "wedge" representation, the initial position of the particle can be taken to be $\left(x_{0}, y_{0}\right)$ with $x_{0} \rightarrow+\infty$. The initial velocity is $-v \hat{e}_{x}$ and $v>0$. As noted before, the metric is locally flat and the trajectory of the particle is simply $\vec{x}(t)=\left(x_{0}-v t\right) \hat{e}_{x}+y_{0} \hat{e}_{y}$. This is valid until the particle has an angular coordinate $\phi=\pi-\delta / 2$ for $y_{0}>0$ or $-\pi+\delta / 2$ for $y_{0}<0$. At this time the identification of the lines $\phi=\pi-\delta / 2$ and $\phi=-\pi+\delta / 2$ must be used and the trajectory of the particle must be continued across the wedge. This leads to a scattering angle given by

$$
\phi_{s} \equiv \phi_{\text {out }}-\phi_{\text {in }}=\left(n_{s} \delta-\pi\right)\left[\Theta\left(y_{0}\right)-\Theta\left(-y_{0}\right)\right],
$$

where $\Theta\left(y_{0}\right)$ is Heaviside step function and $n_{s}$ is the number of scatterings that the particle undergoes in the wedge representation. In other words, $n_{s}$ is the smallest positive integer such that

$$
-\pi+\frac{\delta}{2} \leqq \phi_{s}(\bmod 2 \pi) \leqq \pi-\frac{\delta}{2} .
$$

This condition tells us that there are critical values of the deficit angle $\delta$ at which $n_{s}$ changes by unity. Introducing $\delta \equiv 4 \pi \eta$, with $0 \leqq \eta \leqq 1 / 2$, Eq. (3.1) can be rewritten as

$$
\frac{1}{2}\left(1+\frac{q}{\eta}\right) \leqq n_{s} \leqq \frac{1}{2}\left(1+\frac{q}{\eta}\right)+\frac{1}{2}\left(\frac{1}{\eta}-2\right),
$$

where $q=0,1,2, \ldots$. The smallest $n_{s}$ satisfying (3.2) is

$$
n_{s}=q+1=1,2,3, \ldots,
$$

and corresponds to

$$
\frac{n_{s}-1}{2 n_{s}-1}<\eta \leqq \frac{n_{s}}{2 n_{s}+1} .
$$

This equation tells us that for deficit angles between 0 and $4 \pi / 3$ the particle will be scattered once, twice for $\delta$ between $4 \pi / 3$ and $8 \pi / 5$, and so on. Note that this solution does not depend on the impact parameter in any way. This will not remain true when we consider Coulomb scattering.

Next consider a continuous stream of particles with various impact parameters scattering off a cone with $\delta<4 \pi / 3$. In the region $-\pi+\delta \leqq \phi \leqq \pi-\delta$ (Region I in Fig. 1), the particles are all moving in the $-x$-direction as prescribed by the incoming state. In the region $\pi-\delta \leqq \phi \leqq \pi-\delta / 2$ (Region II), however, there are two streams of particles. One of these streams arises due to the particles that started with $y_{0}>0$, and the other stream is due to the particles that started with $y_{0}<0$ but got scattered into Region II by the cone. This means that the number density 


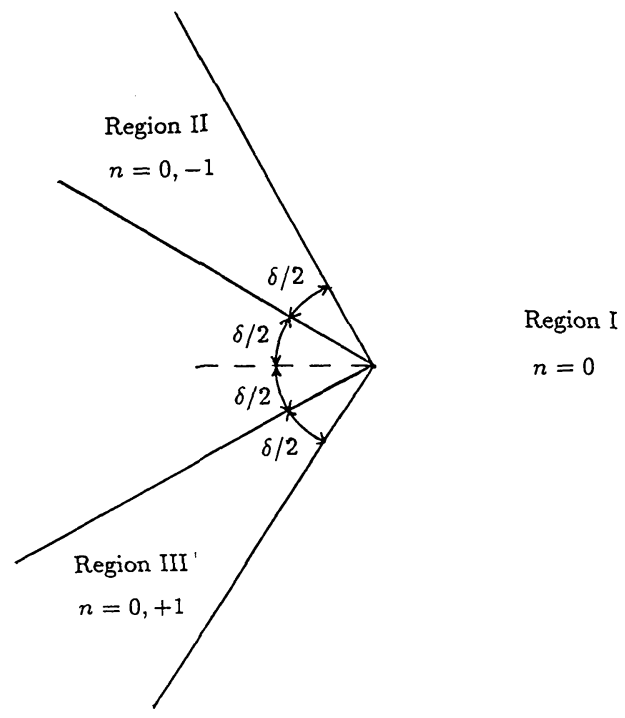

Fig. 1. Wake structure for deficit angle $\delta=\pi / 3$ in absence of potential

of particles in Region II is twice that in Region I. Similar considerations apply to Region III given by $-\pi+\delta / 2 \leqq \phi \leqq-\pi+\delta$. The overdense wedge-like region formed by Regions II and III is known in the cosmic string literature as a "wake" [15].

The structure of the wake depends on the choice of the initial state of the particles. As an example, for cosmic strings moving through a primordial plasma, it seems reasonable to choose the particles to be initially streaming in the $-x$-direction in the rest frame of the string. Although the initial state could be different in other physical problems, we feel that it is most suitable to take the initial state corresponding to the one for cosmic strings. In that case, we should always get a result that exhibits a wake structure. In particular, the quantum mechanical Coulomb scattering should also lead to a wake.

A number of authors $[2,16]$ have considered the quantum mechanical scattering problem on a cone without a potential and with an incoming state

$$
\exp (-i k r \cos (\phi / \alpha))
$$

This incoming state is to be understood as follows: Let us embed the cone in $\mathbb{R}^{3}$ with the $z$-axis as the axis of symmetry of the cone. Then (3.4) represents an incoming wave on the cone that looks like a plane wave when projected on the $x y$-plane.

The local momentum operator in the incoming region on the cone is $\hat{\vec{p}}=-i\left(\partial_{x}, \partial_{y}\right)$, where $(x, y)$ are given by Eq. (2.3). Acting on (3.4) it gives

$$
p_{x}=-k\left[\cos (\phi / \alpha) \cos \phi+\frac{1}{\alpha} \sin (\phi / \alpha) \sin \phi\right] \text {. }
$$

Clearly, (3.4) is not a momentum eigenstate. 
In what follows, we shall always choose the incoming state to be

$$
\exp (-i k r \cos \phi) \text {. }
$$

This initial state can be understood as a plane wave on the cone, after the cone has been flattened out as in the wedge representation. In other words, the wavefronts are straight lines parallel to the $y$-axis in the wedge representation. The local momentum operator acting on (3.5) gives $p_{x}=-k$. Hence (3.5) is indeed an eigenstate of the local momentum operator and corresponds to the incoming state that we have considered in the classical scattering case. This choice also agrees with the choice of 't Hooft [1] and so we can directly compare our results with those of $[1]$.

\section{Coulomb Scattering}

4.1 Classical Scattering. We can discuss the classical Coulomb scattering of a non-relativistic particle by making use of the trajectory given in Eq. (2.2). The asymptotic $(u \rightarrow 0)$ values of $\phi$ are given by $\cos \left(\phi_{\infty}-\phi_{0}\right)= \pm e^{-1}$, where $e=\sqrt{1+4 E^{2} b^{2} / K^{2}}$ is the eccentricity, $b$ is the impact parameter, defined as $j=\mu v b, v$ being the velocity, and $\phi_{0}$ is the angle of the periapsis direction. The plus sign corresponds to the repulsive case, and the minus to the attractive one.

First let us consider the case of an attractive potential $(K>0)$. The scattering angle is defined by $\phi_{s}=2\left(\phi_{\infty}-\phi_{0}\right)+n_{s} \delta-2 \pi$, where $\pi / 2<\phi_{\infty}-\phi_{0}<\pi$. Then,

$$
\phi_{s}=2 \arctan \left(-\frac{2 E b}{K}\right)+n_{s} \delta-2 \pi,
$$

with the understanding that the arc-tangent lies in the second quadrant and we are restricting our attention to $b>0$. As before, $n_{s}$ is the smallest positive integer such that $-\pi+\delta / 2<\phi_{s}(\bmod 2 \pi)<\pi-\delta / 2$. Introducing $2 \pi \beta \equiv 2 \arctan (-2 E b / K)$, with $1 / 2 \leqq \beta \leqq 1$, we have

$$
\frac{q+1-\beta-1 / 2}{2 n_{s}-1} \leqq \eta<\frac{q+1-\beta+1 / 2}{2 n_{s}+1},
$$

where $q$ is a non-negative integer and $\delta \equiv 4 \pi \eta$. Its solution is attained for $q=n_{s}-1$, which corresponds to

$$
\frac{1}{2}-\frac{\beta}{2 n_{s}-1} \leqq \eta<\frac{1}{2}-\frac{\beta}{2 n_{s}+1} .
$$

Notice that now the range of $\eta$ for which we have multiple scattering depends on the impact parameter also. In Fig. 2 we show the regions of the $(\eta, \beta)$ plane that correspond to $n_{s}=1,2$ and 3 . The critical values of $\delta$ for which the number of scatterings changes by one are obtained from (4.1) by taking $\beta=1(b=0)$.

The classical scattering cross-section is given by

$$
\sigma\left(\phi_{s}\right)=\frac{K}{2 E} \sum\left|\frac{d b}{d \phi_{s}}\right|,
$$




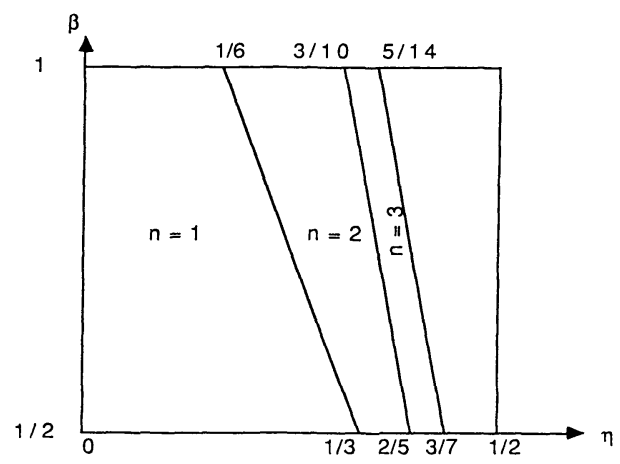

Fig. 2. Values of the deficit angle and the impact parameter for which the particle undergoes single, double and triple classical scattering in an attractive potential

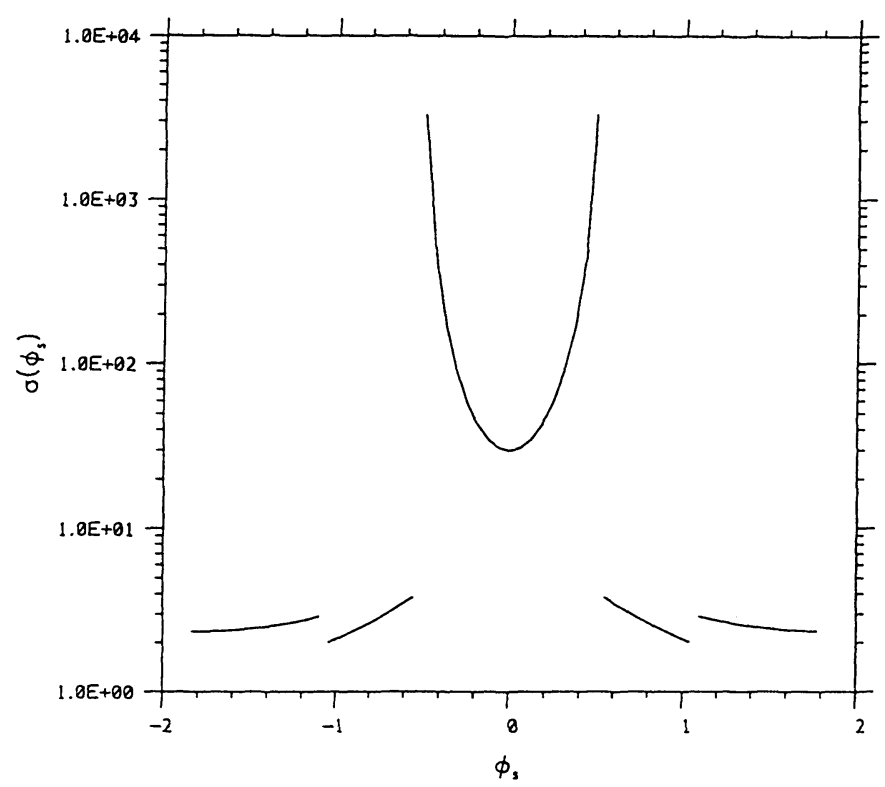

Fig. 3. Classical scattering cross-section for $\delta=5 \pi / 6$ and $K>0$. In plotting we have chosen $K / 4 E=1$

where the sum is over trajectories with $b<0$ and $b>0$. As an example, we plot (Fig. 3) $\sigma\left(\phi_{s}\right)$ versus $\phi_{s}$ for $\phi_{s}<0$ and $\delta=5 \pi / 6$. The discontinuity at $\phi_{s}=-\pi / 3$ is a consequence of the double scattering $\left(n_{s}=2\right)$ of particles with small impact parameters.

We now turn our attention to the case of the repulsive $(K<0)$ Coulomb potential. Once again the scattering angle is

$$
\phi_{s}=2 \arctan \left(-\frac{2 E b}{K}\right)+n_{s} \delta
$$


where the inverse tangent lies in the first quadrant $(b>0)$. Here, too, we find $n_{s}$ by requiring $-\pi+\delta / 2<\phi_{s}(\bmod 2 \pi)<\pi-\delta / 2$. But now $n_{s}=0$ is an acceptable value. We will not discuss the solutions in detail here but will restrict ourselves to a few comments. We find that now $n_{s}=1$ for large $b$ and $n_{s}=0$ for small $b$, if $\delta<4 \pi / 3$ - the critical value in the absence of any Coulomb potential. For $4 \pi / 3<\delta<8 \pi / 5$, the particles with large $b$ get scattered twice $\left(n_{s}=2\right)$ but the particles with small $b$ still undergo single scattering $\left(n_{s}=1\right)$ and the particles with even smaller impact parameter do not get scattered by the wedge $\left(n_{s}=0\right)$. In fact, the particle with zero impact parameter never undergoes any scattering by the wedge. This is just the opposite of the case of the attractive Coulomb potential where we got multiple scattering for zero impact parameter. The critical values for the deficit angle are obtained when the impact parameter is infinite and are the same as for the case of absence of potential.

In the preceding non-relativistic analysis we did not get any "rainbow" scattering or "glory." We have observed that such phenomena do occur when we carry out the relativistic treatment. We plan to study the relativistic particle in a separate publication.

4.2 Quantum Scattering. The non-relativistic quantum scattering is governed by the Schrödinger equation

$$
\left[\frac{1}{r} \frac{\partial}{\partial r}\left(r \frac{\partial}{\partial r}\right)+\frac{1}{r^{2}} \frac{\partial^{2}}{\partial \phi^{2}}+\frac{2 \mu K}{r}+k^{2}\right] \psi_{\alpha}(r, \phi)=0,
$$

where $k^{2} \equiv 2 \mu E$ and $E$ is an arbitrary positive energy. We are interested in finding a solution $\psi_{\alpha}$ to Eq. (4.2) that is regular at the origin, satisfies $\psi_{\alpha}(\phi)=\psi_{\alpha}(\phi+2 \pi \alpha)$ and at infinity can be interpreted as the superposition of an incoming Coulomb wave and a scattered piece. To find such a solution $\psi_{\alpha}$ we use the same method as 't Hooft [1], originally introduced by Sommerfeld [17] to study the classical scattering of electromagnetic waves by a cone. It consists of the following two steps: first solve the scattering problem in the plane with a cut, and second, take care of the periodic boundary condition $\psi_{\alpha}(\phi)=\psi_{\alpha}(\phi+2 \pi \alpha)$.

We will first treat the attractive case, $K>0$.

So then consider Eq. (4.2) in the plane with a branch cut, which we assume to be along the negative real semiaxis, and call its solution $\psi_{c}$. Because we have a cut at $\phi=\pi$, the incoming wave at early times is only defined for $|\phi|<\pi$. It then follows that $\psi_{c}$ has to represent an incoming plane wave as $r \rightarrow \infty$ and $|\phi|<\pi$. It must also reproduce a Coulomb scattered wave as $r \rightarrow \infty$ and $\pi<|\phi| \leqq 2 \pi$. This behaviour can be easily understood using the classical picture: the orbit of a particle approaching the scattering centre is bent because of the attraction of the potential and ultimately will cross the cut onto the next sheet. Now back scattering corresponds to $\phi=|2 \pi|$.

To construct $\psi_{c}$ we start by noticing that

$$
\psi_{1}(r, \phi)=\exp (-i k r \cos \phi) \Phi\left(i Q, \frac{1}{2} ; 2 i k r \cos ^{2}(\phi / 2)\right),
$$

with $\Phi(a, b ; z)$ the confluent hypergeometric function and $Q \equiv \mu K / 2$, is a regular solution to (4.2) for all $r$ and $\phi$. This is precisely the solution for the plane (without 
the cut) and has asymptote

$$
\psi_{1}(r, \phi) \underset{r \rightarrow \infty}{\longrightarrow} \psi_{\text {in }}(r, \phi)+\psi_{\mathrm{sc}}(r, \phi)
$$

where

$$
\psi_{\text {in }}(r, \phi)=\frac{\sqrt{\pi} e^{-\pi Q / 2}}{\Gamma\left(\frac{1}{2}-i Q\right)} \exp \left(-i k r \cos \phi-i Q \ln \left(2 k r \cos ^{2}(\phi / 2)\right)\right)
$$

is an incoming Coulomb wave, and

$$
\psi_{\mathrm{sc}}(r, \phi)=\frac{\sqrt{\pi} e^{-\pi Q / 2}}{\Gamma(i Q)} \frac{\exp \left(i k r+i Q \ln \left(2 k r \cos ^{2}(\phi / 2)\right)\right)}{\sqrt{2 i k r}|\cos (\phi / 2)|}
$$

is an outgoing cylindrical wave with a long range Coulomb correction. Note that the scattered piece has the usual forward $(\phi=\pi)$ singularity, due to the long range nature of the Coulomb potential. It is convenient to write $\psi_{1}$ as

$$
\psi_{1}(r, \phi)=F(r, \phi)+G(r, \phi)
$$

where

$$
\begin{aligned}
& F(r, \phi)=\frac{\sqrt{\pi} e^{-\pi Q}}{\Gamma\left(\frac{1}{2}-i Q\right)} \exp (-i k r \cos \phi) \Psi\left(i Q, \frac{1}{2} ; 2 i k r \cos ^{2}(\phi / 2)\right), \\
& G(r, \phi)=-\frac{i \sqrt{\pi} e^{-\pi Q}}{\Gamma(i Q)} e^{i k r} \Psi\left(\frac{1}{2}-i Q, \frac{1}{2} ;-2 i k r \cos ^{2}(\phi / 2)\right),
\end{aligned}
$$

and $\Psi(a, c ; z)$ is

$$
\Psi(a, c ; z)=\frac{\Gamma(1-c)}{\Gamma(a-c+1)} \Phi(a, c ; z)+\frac{\Gamma(c-1)}{\Gamma(a)} z^{1-c} \Phi(a-c+1,2-c, z) .
$$

The reason for the splitting in (4.6) is that the asymptotes of $F$ and $G$ for large $r$ are precisely $\psi_{\text {in }}$ and $\psi_{\text {sc }}$ :

$$
F(r, \phi) \underset{r \rightarrow \infty}{\longrightarrow} \psi_{\text {in }}(r, \phi), \quad G(r, \phi) \underset{r \rightarrow \infty}{\longrightarrow} \psi_{\text {sc }}(r, \phi)
$$

For $\psi_{c}$ we now write

$$
\begin{aligned}
\psi_{c}(r, \phi)= & \frac{1}{2 \pi i} \int_{C_{1} \cup C_{1}^{\prime}} d z\left[\frac{F(r,-i z)}{z-i \phi}+\frac{G(r,-i z)}{z-i(\phi-2 \pi)}\right] \\
& +\frac{1}{2 \pi i} \int_{C_{2} \cup C_{2}^{\prime}} d z\left[\frac{F(r, i z)}{z-i \phi}+\frac{G(r,-i z)}{z-i(\phi+2 \pi)}\right],
\end{aligned}
$$

where $C_{1}, C_{1}^{\prime}$ and $C_{2}$ and $C_{2}^{\prime}$ are the contours depicted in Fig. 4 . From the fact that $G(r, \phi)$ is periodic in $\phi$ with period $2 \pi$ and that (4.5) solves (4.2) it follows that each of the terms on the right-hand side of (4.8) is a solution of (4.2), and so is (4.8). It still remains to prove that it has the required behaviour at infinity. To do that we re-arrange (4.8) as a sum of integrals around the poles of the integrands and along the horizontal lines of $C_{1}, C_{1}^{\prime}$ and $C_{2}, C_{2}^{\prime}$ : 


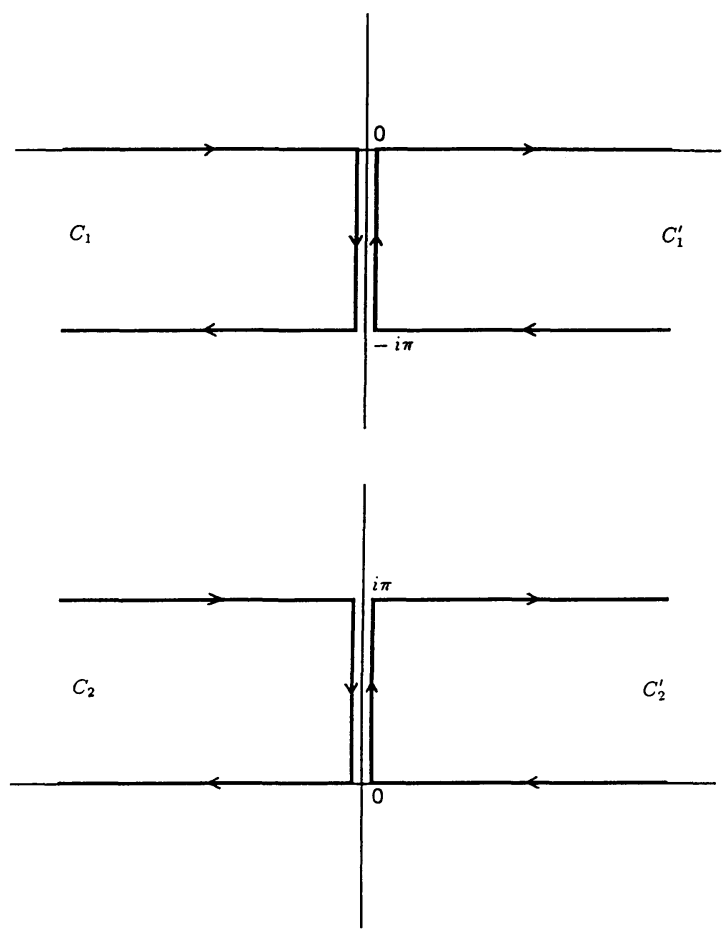

Fig. 4. Contours of integration in (4.16)

$$
\begin{aligned}
\psi_{c}(r, \phi)= & F(r, \phi) \Theta(\pi-|\phi|) \\
& +G(r, \phi)[\Theta(2 \pi-\phi)-\Theta(\pi-\phi)+\Theta(-\pi-\phi)-\Theta(-2 \pi-\phi)] \\
& +\chi_{c}(r, \phi),
\end{aligned}
$$

with

$$
\chi_{c}(r, \phi)=\chi_{1}(r, \phi+\pi)-\chi_{1}(r, \phi-\pi)+\chi_{2}(r, \phi+2 \pi)-\chi_{2}(r, \phi-2 \pi)
$$

and

$$
\begin{aligned}
& \chi_{1}(r, \phi)=\frac{1}{2 \pi i} \int_{-\infty}^{+\infty} \frac{d x}{x+i \phi}[F(r, \pi+i x)-G(r, \pi+i x)], \\
& \chi_{2}(r, \phi)=\frac{1}{2 \pi i} \int_{-\infty}^{+\infty} \frac{d x}{x+i \phi} G(r, 2 \pi+i x) .
\end{aligned}
$$

The asymptotes of the $F$ - and $G$-terms on the right-hand side of (4.9) are already given in (4.7); that of $\chi_{c}$ is (see the Appendix for its derivation)

$$
\begin{aligned}
\chi_{c}(r, \phi) \underset{r \rightarrow \infty}{\longrightarrow} A & \frac{e^{i k r}}{\sqrt{2 i k r}}\left(\frac{1}{\phi-\pi}-\frac{1}{\phi+\pi}\right) \\
& +B \frac{\exp (i k r+i Q \ln (2 k r))}{\sqrt{2 i k r}}\left(\frac{1}{\phi-2 \pi}-\frac{1}{\phi+2 \pi}\right),
\end{aligned}
$$


$A$ and $B$ being $Q$-dependent constants,

$$
A=\frac{e^{\pi Q / 2} \exp (i Q(1-\ln Q))}{\sqrt{2} \Gamma\left(\frac{1}{2}-i Q\right)}, \quad B=\frac{e^{-\pi Q / 2}}{\sqrt{-i Q} \Gamma(i Q)} .
$$

That (4.8) has the required behaviour at large distances is immediate from Eqs. (4.9), (4.7) and (4.13).

Having solved the scattering problem in the plane with a cut it is easy to find the solution for the cone; it is given by

$$
\psi_{\alpha}(r, \phi)=\sum_{n=-\infty}^{\infty} \psi_{c}(r, \phi+2 \pi \alpha n) .
$$

It is a solution of Eq. (4.2) beause it is a sum of solutions, and it obviously satisfies $\psi_{\alpha}(\phi)=\psi_{\alpha}(\phi+2 \pi \alpha)$. It can be written as a contour integral,

$$
\begin{aligned}
\psi_{\alpha}(r, \phi)= & \frac{1}{4 \pi \alpha i} \int_{C_{1} \cup C_{1}^{\prime}} d z\left[\cot \left(\frac{\phi+i z}{2 \alpha}\right) F(r,-i z)+\cot \left(\frac{\phi-2 \pi+i z}{2 \alpha}\right) G(r,-i z)\right] \\
& +\frac{1}{4 \pi \alpha i} \int_{C_{2} \cup C_{2}^{\prime}} d z\left[\cot \left(\frac{\phi+i z}{2 \alpha}\right) F(r,-i z)+\cot \left(\frac{\phi+2 \pi+i z}{2 \alpha}\right) G(r,-i z)\right] .
\end{aligned}
$$

Its asymptote is

$$
\begin{aligned}
\psi_{\alpha}(r, \phi) \underset{r \rightarrow \infty}{\longrightarrow} & \sum_{n=-\infty}^{\infty}\left\{\psi_{\mathrm{in}}\left(r, \phi_{n}\right) \Theta\left(\pi-\left|\phi_{n}\right|\right)\right. \\
& \left.+\psi_{\mathrm{sc}}\left(r, \phi_{n}\right)\left[\Theta\left(2 \pi-\phi_{n}\right)-\Theta\left(\pi-\phi_{n}\right)+\Theta\left(-\pi-\phi_{n}\right)-\Theta\left(-2 \pi-\phi_{n}\right)\right]\right\} \\
& +\frac{A}{2 \alpha} \frac{e^{i k r}}{\sqrt{2 k i r}}\left[\cot \left(\frac{\phi-\pi}{2 \alpha}\right)-\cot \left(\frac{\phi+\pi}{2 \alpha}\right)\right] \\
& +\frac{B}{2 \alpha} \frac{\exp (i k r+i Q \ln (2 k r))}{\sqrt{2 k i r}}\left[\cot \left(\frac{\phi-2 \pi}{2 \alpha}\right)-\cot \left(\frac{\phi+2 \pi}{2 \alpha}\right)\right],
\end{aligned}
$$

where $\phi_{n} \equiv \phi+2 \pi \alpha n$. Equation (4.17) contains an incoming Coulomb wave, $\psi_{\text {in }}\left(r, \phi_{0}\right)$, the standard scattered piece, $\psi_{\mathrm{sc}}\left(r, \phi_{0}\right)$, and many other terms arising due to the topology of the cone.

As a check of consistency let us show that $\psi_{\alpha}$ includes as particular cases the ordinary Coulomb scattering in the plane and the scattering on a cone without potential considered in [1]. The first case corresponds to $\alpha=1$; then from Eqs. (4.15) and (4.9) it follows that $\psi_{\alpha}$ reduces to $\psi_{1}$, which is the solution in the plane. Alternatively, if $\alpha=1$, the last two terms in (4.17) vanish and for the rest we get $\psi_{\text {in }}(r, \phi)+\psi_{\text {sc }}(r, \phi)$, which is the asymptote of the solution for the plane. The second case corresponds to $Q=0$, for which $G(r, \phi)=0$ and (4.16) reduces to 't Hooft's solution, Eq. (5.7) in [1].

If we restrict ourselves to the domain of physical interest, i.e. $-\pi \alpha \leqq \phi \leqq \pi \alpha$, only a finite number of terms enter in the sum of (4.17). This is so because there are finitely many $n$ simultaneously satisfying $-\pi \alpha \leqq \phi \leqq \pi \alpha$ and one (or more) of 


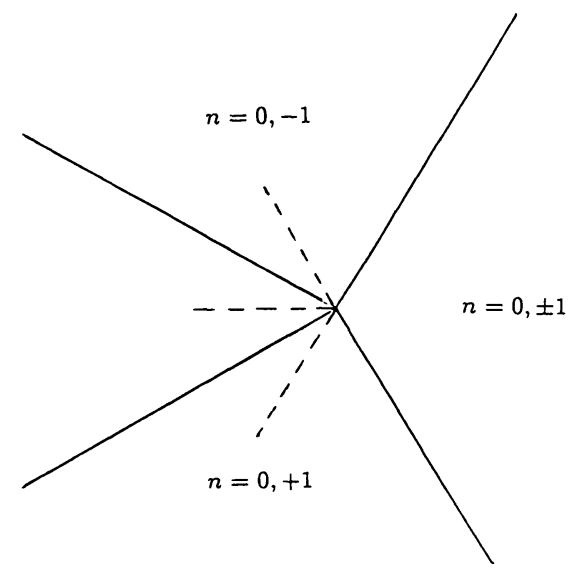

Fig. 5. Wake structure for $\delta=\pi / 3$ in an attractive potential

the conditions $-\pi \leqq \phi+2 \pi \alpha n \leqq \pi,-2 \pi \leqq \phi+2 \pi \alpha n \leqq-\pi, \pi \leqq \phi+2 \pi \alpha n \leqq 2 \pi$ given by the Heaviside functions in (4.17). The allowed values of $n$ depend on the deficit angle and on $\phi$. For $0<\delta<2 \pi / 3$ we can have $n=0, \pm 1$ (see Fig. 5). For $2 \pi / 3 \leqq \delta<6 \pi / 5, n=0, \pm 1, \pm 2$. In general, for $(2 N-1) 2 \pi /(2 N+1) \leqq \delta<$ $(2 N+1) 2 \pi /(2 N+3)$, with $N$ a positive integer there are $2 N+1$ different $n$, $(n=0, \pm 1, \ldots, \pm N)$. We have thus recovered the same sequence of critical values for the deficit angle as in the classical treatment.

Note that not all the terms will contribute to the sum in (4.17) for $\phi$ in the interval $-\pi \alpha \leqq \phi \leqq \pi \alpha$. This is the quantum version of the wake structure discussed in Sect. 3. For example, Fig. 5 shows the regions in which the terms with $n=0, \pm 1$ are defined when $0<\delta<2 \pi / 3$. If we now take $Q=0$ then $\psi_{\mathrm{sc}}=0$ and we only have the conditions given by the $\Theta\left(\pi-\left|\phi_{n}\right|\right)$ in (4.17). So for $0<\delta<2 \pi / 3$ and $Q=0$ instead of Fig. 5 we get Fig. 1, which reproduces explicitly the wake in the absence of potential discussed in Sect. 3. The recovery of the wake depends strongly on the choice of the incoming state, i.e. the fact that in (4.4) one has $\exp (-i k r \cos \phi)$ and not $\exp (-i k r \cos (\phi / \alpha))$, as in [2].

It is interesting to see how the cone has affected the standard singularity of the scattered wave in the forward direction. The forward direction is no longer $\phi=|\pi|$ since it is not in the allowed domain, $-\pi \alpha \leqq \phi \leqq \pi \alpha$. It has been rotated to $\phi=\pi(2 m+1)-2 \pi \alpha n$ for suitable integers $m$ and $n$, where $n$ is the index labelling the terms of the sum in (4.17). For example, for deficit angle $0<\delta<2 \pi / 3, \psi_{\text {sc }}\left(r, \phi_{ \pm 1}\right)$ diverges at $\phi=\mp(\pi+\delta)$.

We next turn our attention to the repulsive case. The analysis is similar to the attractive one. The difference is in the asymptotes that we require for $\psi_{c}$. As in the attractive case, we demand that $\psi_{c}$ represents an incoming plane wave as $r \rightarrow \infty$ and $|\phi|<\pi$. However, we also want it to reproduce the standard Coulomb scattered wave as $r \rightarrow \infty$ and $|\phi|<\pi$. The latter is easy to understand using the classical picture: since the particle is now repelled it will not cross the cut and remain on the same sheet. Back scattering now corresponding to $\phi=0$. Proceeding through 


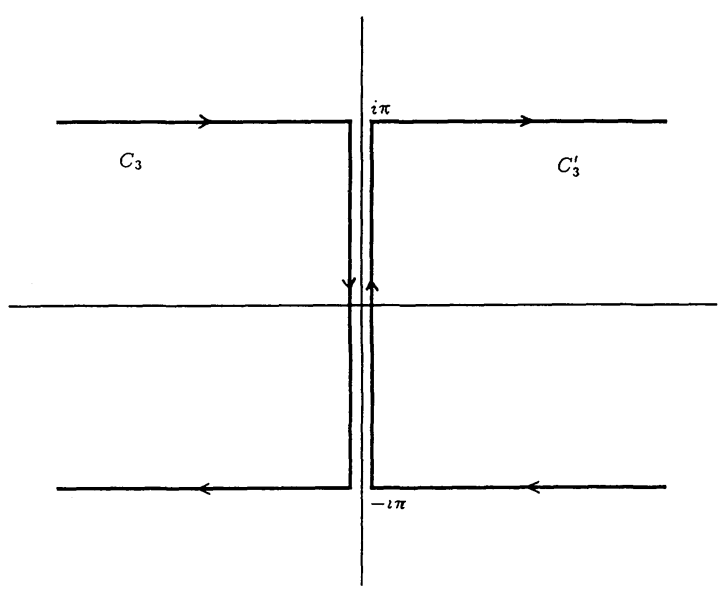

Fig. 6. Contours of integration in (4.19)

the same steps as for the attractive case, one can show that

$$
\psi_{c}(r, \phi)=\frac{1}{2 \pi i} \int_{C_{3} \cup C_{3}^{\prime}} d z \frac{\psi_{1}(r,-i z)}{z-i \phi},
$$

with $C_{3}$ and $C_{3}^{\prime}$ the contours of Fig. 6 , is the solution for the scattering in the plane with a cut. The solution for the cone is given by (4.14) with $\psi_{c}$ as given in (4.18), whose asymptote is

$$
\begin{aligned}
\psi_{\alpha}(r, \phi) \underset{r \rightarrow \infty}{\longrightarrow} & \sum_{n=-\infty}^{\infty}\left[\psi_{\mathrm{in}}\left(r, \phi_{n}\right)+\psi_{\mathrm{sc}}\left(r, \phi_{n}\right)\right] \Theta\left(\pi-\left|\phi_{n}\right|\right) \\
& +\frac{A}{2 \alpha} \frac{e^{i k r}}{\sqrt{2 k i r}}\left[\cot \left(\frac{\phi-\pi}{2 \alpha}\right)-\cot \left(\frac{\phi+\pi}{2 \alpha}\right)\right],
\end{aligned}
$$

which, as before, contains an incoming plane wave, the standard scattered piece and other terms. It is straightforward to check that we can recover the solution for the Coulomb scattering in the plane by taking $\alpha=1$, as well as the scattering on a cone without potential by setting $Q=0$. If we now restrict to angles $-\pi \alpha \leqq \phi \leqq \pi \alpha$ there is a finite number of terms entering in the sum of (4.19). More precisely, for $(N-1) 2 \pi /(2 N-1)<\delta<2 \pi N /(2 N+1)$, with $N$ a positive integer, there are $2 N+1$ permitted values for $n$, namely $n=0, \pm 1, \ldots, \pm N$. This reproduces the same critical deficit angles as in the classical case. The wake structure is now the same as for the case without any potential and differs from the attractive one.

\section{Conclusions}

To summarize, we have studied the motion of non-relativistic particles on a cone with a Coulomb potential. We have found the classical bound orbits and the resulting procession of the perihelion. In the quantum case, the energy levels for the bound states were obtained. It was seen that the degeneracy in the energy 
levels in the planar case (zero deficit angle) was lifted due to the conical geometry. We then studied the scattering problem. The classial scattering gave us the well known wake structure. This wake structure survived in the quantum scattering as well.

\section{Appendix}

In this appendix we derive Eq. (4.13). Taking the limit of large $r$ in (4.11) and (4.12) we obtain

$$
\begin{aligned}
& \chi_{1}(r, \phi) \underset{r \rightarrow \infty}{\longrightarrow} \frac{e^{\pi Q / 2}}{\Gamma\left(\frac{1}{2}-i Q\right)} I(r, \phi)-\frac{e^{-3 \pi Q / 2}}{\Gamma(i Q)} \frac{e^{-i k r}}{\sqrt{-2 i k r}} J(r, \phi), \\
& \chi_{2}(r, \phi) \underset{r \rightarrow \infty}{\longrightarrow} \frac{e^{-\pi Q / 2}}{\Gamma(i Q)} \frac{e^{-i k r}}{\sqrt{2 i k r}} K(r, \phi),
\end{aligned}
$$

where

$$
\begin{aligned}
& I(r, \phi)=\frac{1}{2 \pi i} \int_{-\infty}^{+\infty} \frac{d x}{x+i \phi} \exp \left(i k r \cosh x-i Q \ln \left(2 k r \sinh ^{2}(x / 2)\right)\right) \\
& J(r, \phi)=\frac{1}{2 \pi i} \int_{-\infty}^{+\infty} \frac{d x}{x+i \phi} \frac{\exp \left(i Q \ln \left(2 k r \sinh ^{2}(x / 2)\right)\right)}{|\sinh (x / 2)|} \\
& K(r, \phi)=\frac{1}{2 \pi i} \int_{-\infty}^{+\infty} \frac{d x}{x+i \phi} \frac{\exp \left(i Q \ln \left(2 k r \cosh ^{2}(x / 2)\right)\right)}{|\cosh (x / 2)|} .
\end{aligned}
$$

For $r \rightarrow \infty$ these integrals can be computed, the result being

$$
\begin{aligned}
& I(r, \phi) \underset{r \rightarrow \infty}{\longrightarrow}-\frac{\exp (i Q(1-\ln Q))}{\sqrt{2 \pi}} \frac{e^{i k r}}{\phi \sqrt{2 i k r}}, \\
& J(r, \phi)_{r \rightarrow \infty}^{\longrightarrow}-\frac{2}{\phi} \delta(Q), \\
& K(r, \phi) \underset{r \rightarrow \infty}{\longrightarrow}-\frac{\exp (i Q \ln (2 k r))}{\phi \sqrt{-i \pi Q}} .
\end{aligned}
$$

Combining Eqs. (4.10) and (A.1)-(A.5) we obtain Eq. (4.13). One also has to use that the coefficient in (A.1) multiplying $J$ has a $\Gamma(i Q)$ that, when combined with (A.4), gives no contribution.

Acknowledgements. FRR is grateful to the Spanish Ministry of Education and Science for support through a MEC fellowship. TV was suported by the United Kingdom Science and Engineering Research Council.

\section{References}

1. 't Hooft, G.: Commun. Math. Phys. 117, 685 (1988)

2. Deser, S., Jackiw, R.: Commun. Math. Phys. 118, 495 (1988) 
3. Dowker, J. S.: J. Math. Phys. 30, 770 (1989); Phys. Rev. D36, 3095 (1987)

4. Linet, B.: Phys. Rev. D33, 1833 (1986)

5. Brandenberger, R., Davis, A. C., Matheson, A. M.: Nucl. Phys. B307, 909 (1988)

6. Smith, A. G.: Gravitational effects of an infinite straight cosmic string on classical and quantum fields: self-forces and vacuum fluctuations. TUFTS preprint TUTP-86-11 (1986)

7. Ruback, P. J.: Nucl. Phys. B296, 669 (1988)

8. Ruback, P. J., Shellard, E. P.: Phys. Lett. 209B, 262 (1988)

9. Carlip, S.: Nucl. Phys. B324, 106 (1989)

10. Lagrange, J. L.: Nouveau memoire de l'Academie Royale des Sciences et Belles Lettres de Berlin (1781), in Oeuvres de Lagrange, vol. 5, p. 132

11. Deser, S., Jackiw, R., 't Hooft, G.: Ann. Phys. 152, 220 (1984)

12. Henneaux, M.: Phys. Rev. D29, 2766 (1984)

13. Hankins, T. L.: Sir William Rowan Hamilton. Baltimore, MD: Johns Hopkins University Press 1983

14. Kay, B. S., Studer, U. M.: Quantum mechanics and field theory on cones and cosmic strings. University of Zurich and ETH preprint (1989)

15. Silk, J., Vilenkin, A.: Phys. Rev. Lett. 53, 1700 (1984)

16. de Sousa Gerbert, Ph., Jackiw, R.: Commun. Math. Phys. 124, 229-260 (1989)

17. Sommerfeld, A.: Proc. Lond. Math. Soc. 28, 417 (1897)

Communicated by L. Alvarez-Gaumé

Received June 6, 1989 